\title{
Critérios empiricamente verificáveis para a avaliação do vocabulário na produção oral do professor de língua estrangeira
}

\section{Empirically verifiable criteria to assess vocabulary accuracy in the oral production of foreign language teachers}

Diego Fernando de OLIVEIRA*

\begin{tabular}{|c|c|}
\hline $\begin{array}{l}\text { RESUMO: O EPPLE (Exame de } \\
\text { Proficiência para Professores de Línguas } \\
\text { Estrangeiras) é um instrumento avaliativo } \\
\text { em constante aprimoramento } \\
\text { (CONSOLO; TEIXEIRA DA SILVA, 2014; } \\
\text { COLOMBO, 2019) que se volta para a } \\
\text { avaliação da proficiência do professor de } \\
\text { LE no contexto brasileiro. Atualmente, o } \\
\text { EPPLE possui apenas uma escala holística } \\
\text { para a avaliação do desempenho oral de } \\
\text { candidatos, desenvolvida intuitivamente. } \\
\text { Dessa forma, é necessário que se construa } \\
\text { uma escala de proficiência analítica } \\
\text { empiricamente embasada para a } \\
\text { produção de um argumento para a } \\
\text { validade dos critérios avaliativos que } \\
\text { classificam os desempenhos orais no } \\
\text { exame. A precisão do vocabulário } \\
\text { empregado, entre outros construtos da } \\
\text { competência linguística, é de extrema } \\
\text { importância para a proficiência oral do } \\
\text { professor de LE, uma vez que o professor } \\
\text { ensina a LE utilizando a língua-alvo em } \\
\text { contextos comunicativos (FREEMAN et } \\
\text { al., 2015). O presente estudo apresenta } \\
\text { resultados da aplicação dos EBBs } \\
\text { (Empirically derived, Binary choice, }\end{array}$ & $\begin{array}{l}\text { ABSTRACT: The EPPLE (Proficiency } \\
\text { Exam for Foreign Language Teachers) is } \\
\text { an assessment instrument in constant } \\
\text { improvement (CONSOLO; TEIXEIRA } \\
\text { DA SILVA, 2014; COLOMBO, 2019) } \\
\text { which aims at assessing the proficiency of } \\
\text { the FL teacher in the Brazilian context. } \\
\text { Currently, the EPPLE has only a holistic } \\
\text { scale to assess the oral performance of } \\
\text { candidates, developed intuitively. Thus, } \\
\text { it is necessary to build an analytical and } \\
\text { empirically based proficiency scale to } \\
\text { produce an argument for the validity of } \\
\text { the assessment criteria that classify the } \\
\text { oral performances in the exam. The } \\
\text { vocabulary accuracy, among other } \\
\text { constructs of the linguistic competence, is } \\
\text { extremely important for the FL language } \\
\text { teacher oral proficiency, since the teacher } \\
\text { teaches LE using the target language in } \\
\text { communicative contexts (FREEMAN et } \\
\text { al., 2015). The present study presents } \\
\text { results of the application of EBBs } \\
\text { (Empirically derived, Binary choice, } \\
\text { Boundary definition scales), an empirical } \\
\text { methodology for the development of } \\
\text { assessment criteria and rating scales }\end{array}$ \\
\hline
\end{tabular}

\footnotetext{
* Mestrando no programa de Pós-Graduação em Estudos Linguísticos da UNESP/IBILCE. Orcid: https://orcid.org/0000-0002-5121-6237. Email: diego.fernando@unesp.br.
} 


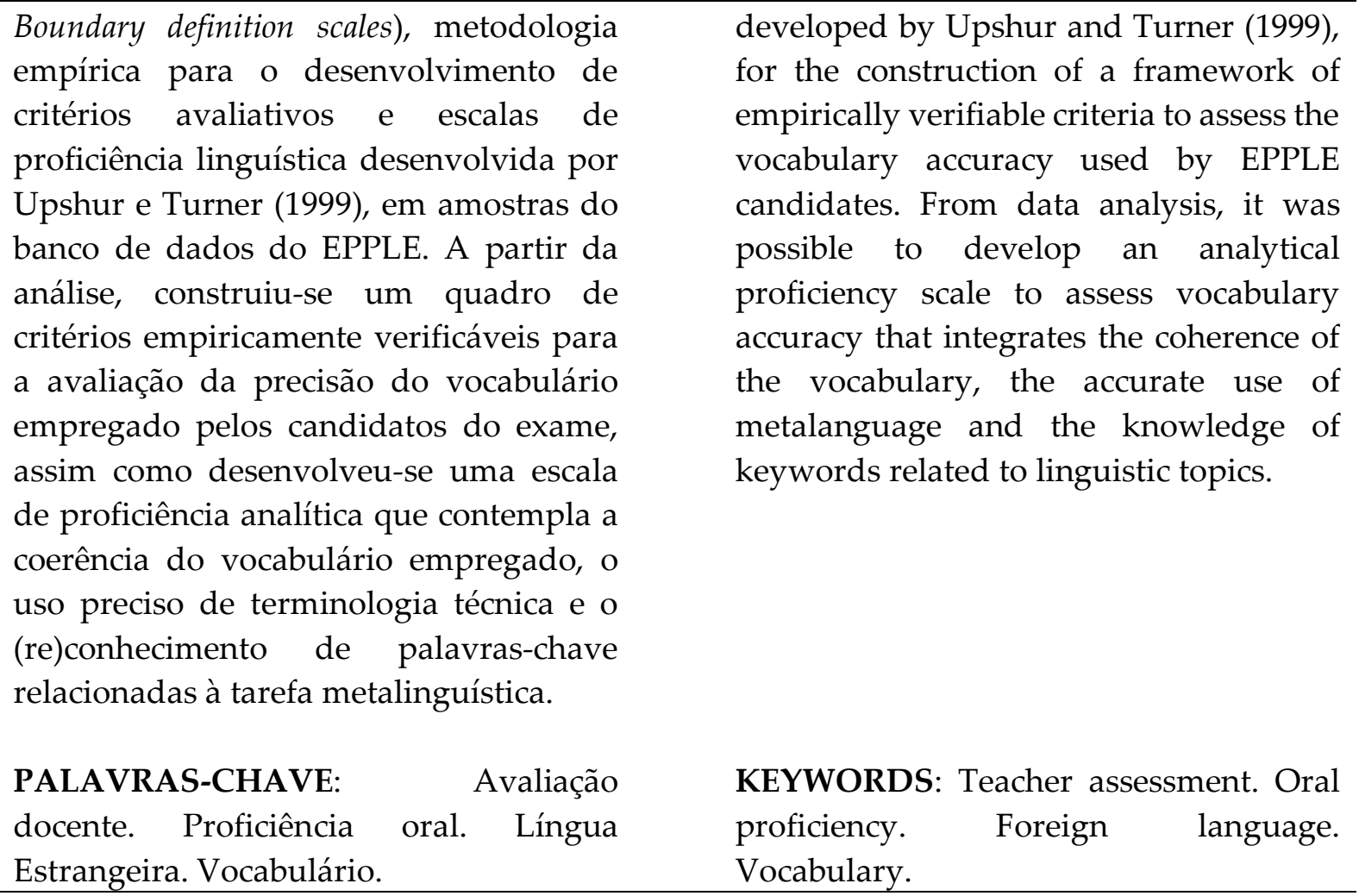

\section{Introdução}

O ensino de línguas estrangeiras (doravante LE) no contexto brasileiro apresenta um histórico caracterizado por uma série de fatores desfavoráveis para o desenvolvimento da proficiência linguística entre professores e alunos. No final do século XX, Almeida Filho (1992) apresentava indícios de baixos níveis de proficiência entre professores de LE que atuavam na educação básica no contexto nacional, assim como Nicholls (2001), no início deste século, também discutia aspectos deficitários na produção oral do professor de LE no Brasil. Ainda na mesma década, Sandei (2005) discorria sobre os baixos níveis de proficiência em língua inglesa entre alunos da educação básica brasileira.

Mais recentemente, Rodrigues (2016), Consolo (2017) e Colombo (2019) discutem em seus trabalhos a baixa proficiência oral do professor de LE e questões relacionadas à sua formação nos cursos de Licenciatura em Letras. Para Consolo (2017), por exemplo, é possível observar que, no contexto brasileiro, há uma série de 
fatores que atuam para a manutenção de um ciclo de baixo nível de proficiência linguística que afeta tanto o ensino básico quanto o ensino superior - nesse caso, especificamente, os cursos de formação de professores de LEs.

Pensando nesse contexto, o EPPLE (Exame de Proficiência para Professores de Línguas Estrangeiras), instrumento avaliativo específico para professores de LEs, é um exame em desenvolvimento e em constante aprimoramento (CONSOLO; TEIXEIRA DA SILVA, 2014; CONSOLO; AGUENA, 2017; CONSOLO, 2017; COLOMBO, 2019) que visa à coleta de dados acerca do desempenho oral e escrito do professor de LE. Espera-se que esses dados possam orientar futuras reelaborações nas políticas educacionais dos cursos de Licenciatura em Letras e, em um cenário otimista, contribuir para a reversão de um ciclo deficitário para o ensino de LEs no Brasil.

Um elemento importante do construto da proficiência oral do professor e, nesse caso, da competência linguística, é o vocabulário (FULCHER, 2014). A precisão do vocabulário empregado pelo professor de LE é extremamente importante para prover feedback aos alunos e abordar problemas linguísticos utilizando metalinguagem, assim como outras áreas funcionais específicas da produção oral docente (ELDER, 2001; FREEMAN et al., 2015). Dessa forma, é importante desenvolver um quadro de critérios válidos para a avaliação do vocabulário na produção oral docente para que se possa obter resultados confiáveis através da aplicação do EPPLE.

Dessa forma, este artigo pretende contribuir com o aprimoramento do construto do EPPLE, sugerindo uma escala de proficiência analítica voltada para a avaliação da precisão do vocabulário empregado na tarefa metalinguística do exame. Além disso, objetiva-se verificar questões levantadas por Colombo (2019) acerca de inconsistências presentes nos descritores da escala de proficiência holística utilizada para a classificação dos desempenhos orais dos candidatos do exame. Por fim, o presente trabalho pretende contribuir com o desenvolvimento de um argumento para a 
validade do construto do EPPLE, uma vez que visa apresentar um quadro de critérios passível de verificação empírica.

\section{O EPPLE e a avaliação da proficiência oral do professor de LE}

O EPPLE é um exame de proficiência em desenvolvimento que objetiva a avaliação da proficiência oral e escrita de professores de LEs em formação e em atuação (COLOMBO, 2019). Em sua versão oral, ele é divido em cinco tarefas, sendo a tarefa número cinco responsável por estimular a produção oral para a avaliação do conhecimento específico do candidato acerca de um tópico linguístico, assim como do emprego de metalinguagem, ambas áreas funcionais da proficiência oral intrínsecas à atividade docente (ELDER, 2001; FREEMAN et al., 2015).

A Figura 1, a seguir, apresenta o diagrama desenvolvido por Freeman et al. (2015) que ilustra o construto da proficiência oral do professor de LE, assim como as áreas funcionais da produção oral docente.

Figura 1 - Diagrama do construto da proficiência do professor de LE e suas áreas funcionais, baseado em Freeman et al. (2015).

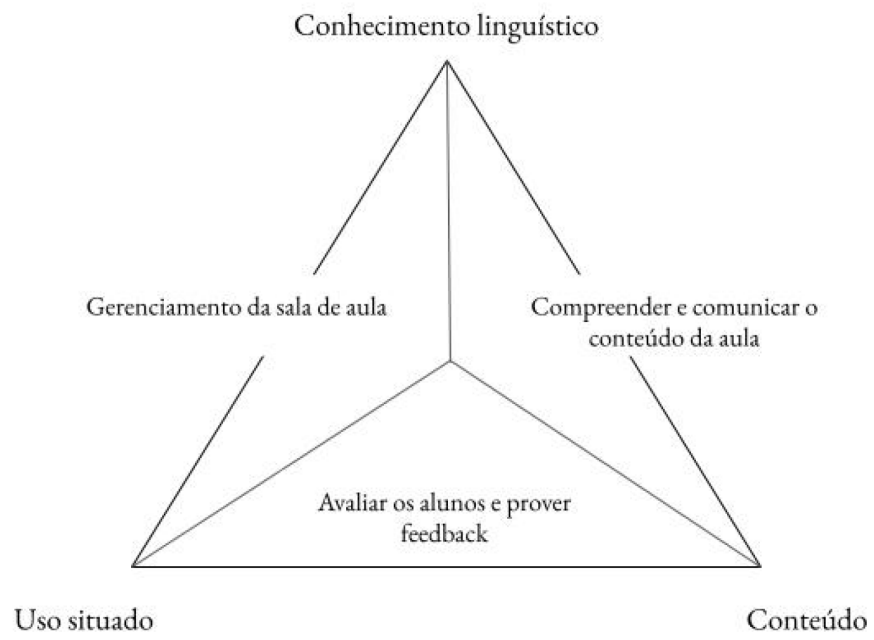

Fonte: o autor.

No diagrama, é possível verificar que o construto do professor de LE é composto por um tripé: o conhecimento linguístico necessário para o ensino da LE, o 
uso situado que se relaciona ao contexto específico de ensino da LE na língua-alvo e, por fim, o conteúdo que se refere a tópicos linguísticos a serem abordados durante a aula. Dessa forma, é possível verificar que o gerenciamento da sala, a compreensão e comunicação do conteúdo da aula, assim como avaliação e provimento de feedback resultam da intersecção das três diferentes dimensões do construto da proficiência oral docente.

Dessa maneira, atentando-se às especificidades da produção oral do professor de LE, a tarefa número cinco do EPPLE apresenta tópicos linguísticos em forma de dúvidas apresentadas por alunos, a fim de que o candidato possa prover explicações, assim como possa empregar metalinguagem para abordar as situações propostas. $\mathrm{O}$ Excerto 1, a seguir, apresenta os tópicos linguísticos propostos aos candidatos pelo EPPLE em sua versão 3.1.

\section{Situação A}

Excerto 1 - Tópicos linguísticos propostos na versão 3.1 do EPPLE.

I don't know if these sentences are correct. The first is "If I happen to run into her, I would tell her where you live". The second is "If antibiotic drugs like peniciline were available during World War I, many more lives could be saved". Could you help me?

\section{Situação B}

These sentences are really confusing in this exercise. Which alternative best completes the sentence: "I've written to Maria but she hasn't replied?" Letter A: "She might be away. You never know with her". Letter B: "She must be in Salvador. You never know". How can you explain that?

Fonte: Colombo (2019).

Para Colombo (2019), os critérios que orientam a avaliação do vocabulário empregado pelos candidatos em suas explicações no EPPLE podem ter sua validade questionada, uma vez que permitem a classificação de candidatos que expressam desconhecimento sobre os tópicos linguísticos nas faixas superiores apenas por 
empregarem terminologia técnica. Dessa maneira, para a autora, é necessário que se revisem os descritores da escala de proficiência holística do exame, a fim de que a precisão do vocabulário empregado no provimento de explicações e no uso da metalinguagem seja contemplada.

\section{Critérios para a avaliação do vocabulário}

Para a avaliação do vocabulário na produção oral, é necessário delimitar uma área de interesse que melhor defina os aspectos relevantes para o construto que nos interessa avaliar. Primeiramente, é possível dividir vocabulário em dois âmbitos diferentes: abrangência e profundidade ${ }^{1}$. Milton (2009) define abrangência como o número total de palavras que um aprendiz de LE sabe e profundidade como o que o aprendiz sabe sobre essas palavras, ou seja, sobre seu significado, sobre sua adequação e sobre sua ocorrência típica em enunciados. Além disso, segundo o autor, a avaliação do vocabulário pode considerar outra convenção: dividir o conhecimento do vocabulário em conhecimento passivo (ou receptivo) e produtivo (ou ativo) (MILTON, 2009).

A Figura 2, a seguir, ilustra a relação entre a abrangência do vocabulário e a sua respectiva profundidade no desempenho oral. Tal relação também é referida na literatura como espaço lexical ${ }^{2}$ (DALLER et al., 2007). Dessa maneira, é possível observar na figura que, quanto maior a abrangência e a profundidade do vocabulário, maior tende a ser a fluência da produção oral em LE.

\footnotetext{
${ }^{1}$ No original: Beadth e Depth, Milton (2009).

2 No original: Lexical space, Daller et al. (2007).
} 
Figura 2 - Dimensões do conhecimento de vocabulário, baseado em Daller et al. (2007).

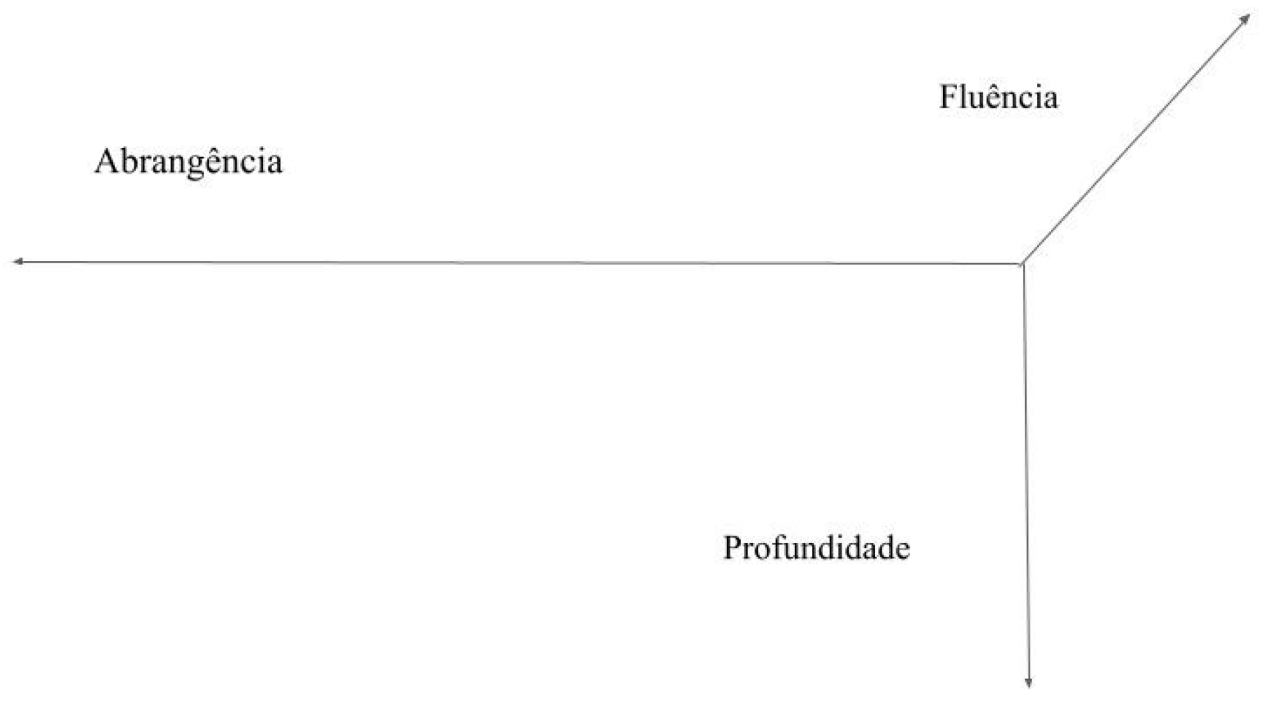

Fonte: o autor.

Na produção oral, é através do conhecimento receptivo que as palavras são reconhecidas quando ouvidas e através de seu conhecimento produtivo é que palavras podem ser mentalmente evocadas e utilizadas em enunciados. Além de tais delimitações teóricas para se avaliar e investigar o vocabulário dentro do construto da proficiência oral, é preciso considerar, ainda, a definição de palavra em si principalmente nos casos em que se deseja investigar a abrangência do vocabulário. Para Milton (2009),

Não há uma única, simples definição de uma palavra que é utilizada na criação de testes para mensurar conhecimento de vocabulário e aprendizagem. Investigadores podem mudar a unidade de mensuração de acordo com as circunstâncias e os aprendizes que eles estão investigando ${ }^{3}$ (MILTON, 2009, p. 12).

\footnotetext{
${ }^{3}$ No original: There is no single, simple definition of a word that is used in the creation of tests to measure vocabulary knowledge and learning. Investigators can change the unit of measurement according to the circumstances and the learners they are investigating (MILTON, 2009, p. 12).
} 
Considerando a necessidade de se estabelecer recortes específicos os quais devem contemplar qual aspecto do construto da proficiência oral e do conhecimento do vocabulário se tem o interesse de investigar, o Quadro 1, a seguir, desenvolvido por Nation (2001), apresenta possíveis definições operacionais para a avaliação do vocabulário na produção oral, considerando tanto o conhecimento quanto o conhecimento passivo.

No Quadro 1, divide-se o construto do conhecimento de vocabulário em três diferentes áreas de interesse: (i) Forma, que se relaciona à estrutura do vocabulário em si, como ele se parece em forma escrita ou como ele soa; (ii) Significado, que se relaciona à identificação de uma forma e sua associação a um ou mais significados; (iii) Uso, que se define pela relação que uma palavra estabelece com outras em um enunciado. Tais divisões possuem subcategorias para a língua escrita e língua falada e, por fim, são fracionadas em conhecimento receptivo $(\mathrm{R})$ e conhecimento produtivo $(\mathrm{P})$.

Quadro 1 - Possíveis critérios para a avaliação do vocabulário, segundo Nation (2001).

\begin{tabular}{|c|c|c|c|}
\hline \multirow[t]{6}{*}{ Forma } & \multirow[t]{2}{*}{ Falado } & $\mathrm{R}$ & Como a palavra soa? \\
\hline & & $\mathrm{P}$ & Como a palavra é pronunciada? \\
\hline & \multirow[t]{2}{*}{ Escrito } & $\mathrm{R}$ & Como a palavra se parece? \\
\hline & & $\mathrm{P}$ & Como a palavra é escrita? \\
\hline & \multirow[t]{2}{*}{ Partes das palavras } & $\mathrm{R}$ & $\begin{array}{l}\text { Quais partes são reconhecíveis nessa } \\
\text { palavra? }\end{array}$ \\
\hline & & $\mathrm{P}$ & $\begin{array}{llr}\text { Quais partes } & \text { das palavras são } \\
\text { necessárias } & \text { para } & \text { expressar } \\
\text { significado? } & & \end{array}$ \\
\hline \multirow[t]{3}{*}{ Significado } & \multirow[t]{2}{*}{ Forma e significado } & $\mathrm{R}$ & $\begin{array}{l}\text { Qual o significado indicado / sugerido } \\
\text { pela forma desta palavra? }\end{array}$ \\
\hline & & $\mathrm{P}$ & $\begin{array}{l}\text { Qual forma lexical pode ser utilizada } \\
\text { para expressar esse significado? }\end{array}$ \\
\hline & Conceitos e referentes & $\mathrm{R}$ & O que é incluído nesse conceito? \\
\hline
\end{tabular}




\begin{tabular}{|c|c|c|c|}
\hline & & $\mathrm{P}$ & $\begin{array}{l}\text { A quais itens o conceito pode se } \\
\text { referir? }\end{array}$ \\
\hline & \multirow[t]{2}{*}{ Associações } & $\mathrm{R}$ & $\begin{array}{l}\text { Em quais outras palavras essa palavra } \\
\text { nos faz pensar? }\end{array}$ \\
\hline & & $\mathrm{P}$ & $\begin{array}{l}\text { Quais outras palavras podemos usar } \\
\text { ao invés dessa? }\end{array}$ \\
\hline \multirow[t]{6}{*}{ Uso } & \multirow[t]{2}{*}{ Funções gramaticais } & $\mathrm{R}$ & Em quais padrões a palavra ocorre? \\
\hline & & $\mathrm{P}$ & $\begin{array}{l}\text { Em quais padrões nós devemos usar } \\
\text { essa palavra? }\end{array}$ \\
\hline & \multirow[t]{2}{*}{ Colocações } & $\mathrm{R}$ & $\begin{array}{l}\text { Quais palavras ou tipos de palavras } \\
\text { ocorrem com essa? }\end{array}$ \\
\hline & & $\mathrm{P}$ & $\begin{array}{l}\text { Quais palavras ou tipos de palavras } \\
\text { nós devemos usar com essa? }\end{array}$ \\
\hline & \multirow[t]{2}{*}{ Restrições de uso } & $\mathrm{R}$ & $\begin{array}{l}\text { Onde, quando e com que frequência } \\
\text { deveríamos encontrar essa palavra? }\end{array}$ \\
\hline & & $\mathrm{P}$ & $\begin{array}{l}\text { Onde, quando e com que frequência } \\
\text { podemos usar essa palavra? }\end{array}$ \\
\hline
\end{tabular}

Fonte: Nation (2001, p. 27).

Dessa forma, Nation (2001) apresenta um quadro de possíveis definições operacionais que auxilia avaliadores, pesquisadores e construtores de testes a delimitar o foco investigativo e os aspectos-chave para a avaliação do conhecimento de vocabulário na produção oral. Além de tais especificações, Read (2000) destaca três dimensões da avaliação do vocabulário que devem ser levadas em consideração, uma vez que são importantes para a definição operacional de um teste ou exame, assim como para a validade de seus critérios avaliativos. O Quadro 2, a seguir, apresenta as três dimensões da avaliação do vocabulário, segundo Read (2000). 
Quadro 2 - Dimensões da avaliação do vocabulário.

\begin{tabular}{|l|l|}
\hline $\begin{array}{l}\text { Discreto } \\
\text { como um construto independente. }\end{array}$ & $\begin{array}{l}\text { Integrado } \\
\text { Mensura o vocabulário como um componente } \\
\text { de um construto maior. }\end{array}$ \\
\hline $\begin{array}{l}\text { Seletivo } \\
\text { Mensura o foco da avaliação. }\end{array}$ & $\begin{array}{l}\text { Compreensivo } \\
\text { Mensura o vocabulário considerando todo o } \\
\text { conteúdo presente no input (tarefas de } \\
\text { leitura/compreensão auditiva) ou na resposta } \\
\text { do candidato (escrever/falar). }\end{array}$ \\
\hline $\begin{array}{l}\text { Independente do contexto } \\
\text { Uma mensuração do vocabulário que considera a a } \\
\text { resposta do candidato que não se refere a um } \\
\text { contexto específico. }\end{array}$ & $\begin{array}{l}\text { Dependente do contexto } \\
\text { habilidade do candidato de considerar } \\
\text { informação contextual para a produção da } \\
\text { resposta. }\end{array}$ \\
\hline
\end{tabular}

Fonte: Read (2000, p. 9).

Na primeira dimensão, o vocabulário pode ser considerado parte de um construto maior ou ser avaliado como um construto independente, separado de outras competências linguísticas. Na segunda dimensão, leva-se em conta a abrangência do número de palavras contempladas na avaliação do vocabulário, ou seja, se há delimitação de algumas palavras de interesse do avaliador ou se todas as palavras produzidas serão consideradas na avaliação. Por fim, na terceira dimensão, o vocabulário pode ser avaliado como independente ou dependente de um contexto avaliativo específico.

Por fim, é importante mencionar os critérios presentes no CEFR (Common European Framework) para a avaliação do vocabulário. Divididas em diferentes categorias, critérios para a avaliação da abrangência do vocabulário e de sua precisão podem ser encontradas nas escalas Linguistic Range, Vocabulary Range e Vocabulary Control. O Quadro 3, a seguir, apresenta um resumo dos critérios utilizados para a classificação dos desempenhos satisfatórios e insatisfatórios nas escalas de proficiência linguística mencionadas. 
Quadro 3 - Critérios para a avaliação do vocabulário no CEFR.

\begin{tabular}{|c|c|}
\hline Escala de proficiência & Critérios \\
\hline \multirow{2}{*}{ Linguistic Range } & $\begin{array}{l}\text { Satisfatórios: O candidato possui amplo vocabulário } \\
\text { empregado precisamente, assim como pode } \\
\text { diferenciar e desambiguizar palavras. Não } \\
\text { demonstra ter limitações de vocabulário. }\end{array}$ \\
\hline & $\begin{array}{l}\text { Insatisfatórios: O candidato possui vocabulário } \\
\text { suficiente para se expressar em contextos específicos. } \\
\text { Demonstra limitações de vocabulário, apresenta } \\
\text { repetição de palavras ao tratar de temas comuns, } \\
\text { assim como busca mentalmente por palavras, } \\
\text { expressões básicas do dia a dia etc. }\end{array}$ \\
\hline \multirow[t]{2}{*}{ Vocabulary Range } & $\begin{array}{l}\text { Satisfatório: O candidato possui amplo repertório, } \\
\text { expressões idiomáticas, coloquialismo, conotação. } \\
\text { Supre falta de vocabulário suprido por paráfrases. } \\
\text { Faz poucas buscas mentais por palavras. Conhece } \\
\text { sinônimo de palavras pouco comuns. Usa amplo } \\
\text { vocabulário técnico de sua área de conhecimento. }\end{array}$ \\
\hline & $\begin{array}{l}\text { Insatisfatório: Evita repetições frequentes, há } \\
\text { hesitação e paráfrase na falta de uma palavra. Tem } \\
\text { problemas com termos técnicos fora de sua área. } \\
\text { Vocabulário necessário para tratar de temas do dia a } \\
\text { dia. }\end{array}$ \\
\hline \multirow[t]{2}{*}{ Vocabulary Control } & $\begin{array}{l}\text { Satisfatório: Uso apropriado de palavras menos } \\
\text { comuns (às vezes com erros não significativos), } \\
\text { ocorrem escolhas incorretas sem que a compreensão } \\
\text { seja afetada. }\end{array}$ \\
\hline & $\begin{array}{l}\text { Insatisfatório: Comete erros mais graves ao falar de } \\
\text { temas pouco familiares, mais complexos. Controla } \\
\text { vocabulário simples que se refere ao dia a dia. }\end{array}$ \\
\hline
\end{tabular}

Fonte: o autor.

É importante ressaltar que o quadro fornece apenas um resumo dos critérios presentes nas escalas do CEFR, os quais podem ser consultados integralmente no website do Conselho Europeu ${ }^{4}$. Além disso, foram categorizados como satisfatórios os

\footnotetext{
${ }^{4}$ Disponível em: https://rm.coe.int/common-european-framework-of-reference-for-languages-learningteaching/16809ea0d4
} 
critérios presentes nas faixas mais altas das escalas de proficiência, assim como foram categorizados como insatisfatórios os critérios presentes nas faixas mais baixas das escalas mencionadas. Dessa forma, é possível ter uma visão mais global dos critérios utilizados para a avaliação do vocabulário na produção oral em exames de proficiência, uma vez que o CEFR é uma referência importante para a avaliação de proficiência oral e escrita em LE.

\section{Procedimentos metodológicos}

Para a análise dos dados, uma coleção de vinte e oito amostras de desempenho oral oriundas do banco de dados do EPPLE foi utilizada, compreendendo amostras coletadas nos anos de 2015 e 2017 em universidades públicas do estado de São Paulo e Minas Gerais ${ }^{5}$. As amostras consistem em gravações de produções orais de licenciandos em Letras que realizaram o EPPLE. Além disso, para este trabalho, foram consideradas apenas as respostas providas à tarefa metalinguística do exame, uma vez que a metodologia empregada produz critérios estritamente relacionados a uma única tarefa (FULCHER et al., 2011).

É importante destacar que as amostras de desempenho oral foram transcritas com a utilização do software CLAN ${ }^{6}$. O CLAN permite a transcrição de gravações em formato de diálogos e possui códigos próprios para codificar diferentes fenômenos da produção oral. O Anexo 1, ao fim deste artigo, apresenta os códigos empregados nas transcrições dos desempenhos orais e seus respectivos significados.

Os EBBs (Empirically derived, Binary choice, Boundary definition scales) é uma metodologia empírica para o desenvolvimento de critérios avaliativos e escalas de proficiência linguística desenvolvida por Upshur e Turner (1999) que consiste no

\footnotetext{
${ }^{5}$ Número do protocolo do processo do Comitê de Ética da UNESP/IBILCE: 0007.0.229.325-11.

${ }^{6}$ Disponível em https://talkbank.org/software/
} 
desenvolvimento de Árvores de Decisão de Desempenho ${ }^{7}$. Através de uma série de perguntas binárias (sim/não), desenvolve-se uma Árvore de Decisão de Desempenho que irá compor, ao final do processo, uma escala de proficiência linguística empiricamente verificável que estabelece limites precisos entre as diferentes faixas de proficiência produzidas.

A Figura 3, a seguir, ilustra o desenvolvimento de uma Árvore de Decisão de Desempenho e de uma escala de proficiência linguística de quatro níveis a partir da aplicação dos EBBs.

Figura 3 - Esquema de desenvolvimento de Árvores de Decisão de Desempenho e escalas de proficiência linguística através dos EBBs.

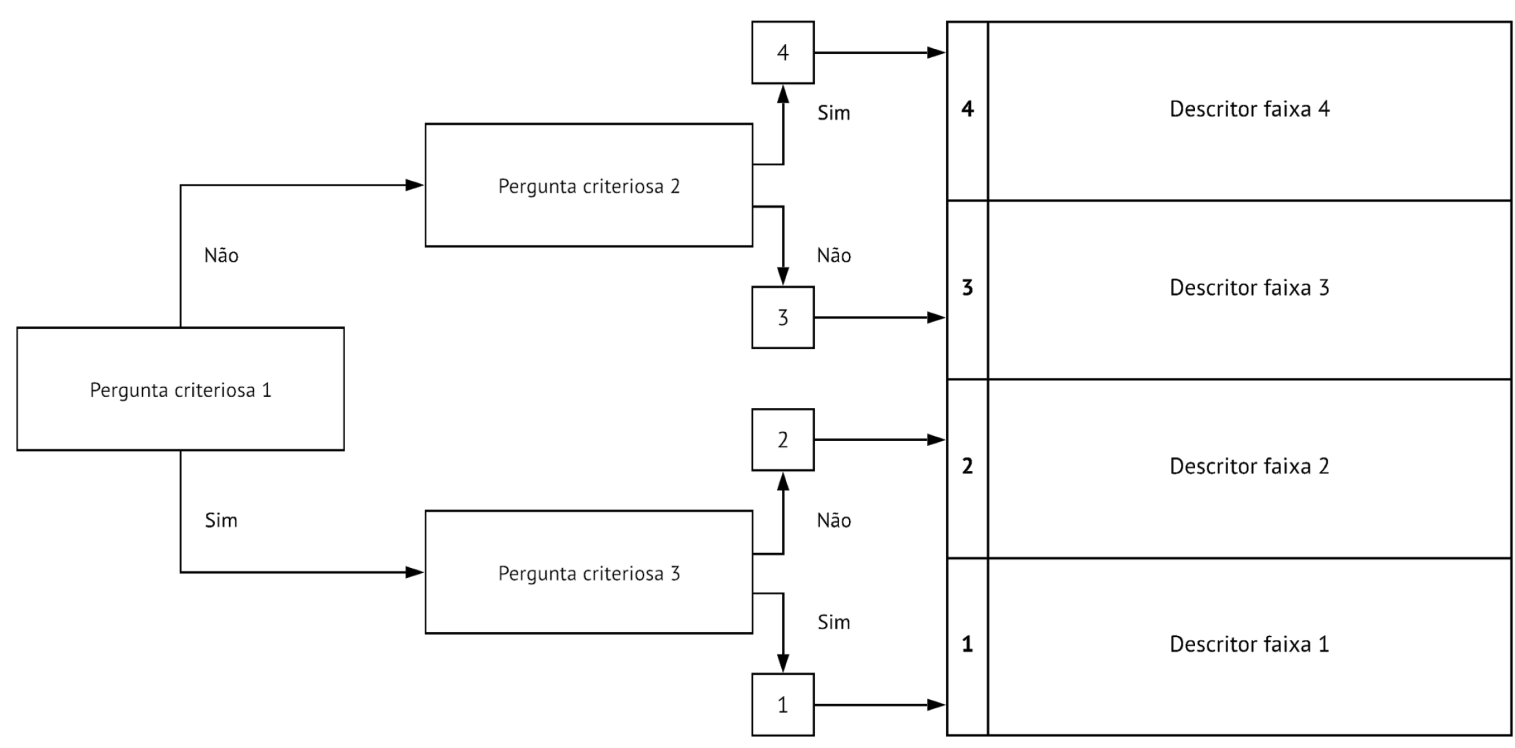

Fonte: o autor.

Dessa forma, como é possível observar, a Pergunta Criteriosa 1 estabelece os limites entre os dois níveis mais altos e mais baixos da escala - ou seja, classifica os desempenhos mais e menos satisfatórios observados nos dados. A Pergunta Criteriosa 2 divide os desempenhos satisfatórios, caracterizando os desempenhos mais proficientes entre as amostras analisadas. O mesmo acontece com a Pergunta

\footnotetext{
${ }^{7}$ No original: Performance Decision Trees (FULCHER et al., 2011).
} 
Criteriosa 3, a qual distingue os desempenhos menos satisfatórios e caracteriza o desempenho menos proficiente observado nas amostras analisadas.

\section{Análise de dados}

A partir da análise das amostras de desempenho oral oriundas do banco de dados do EPPLE, foi possível desenvolver um quadro de critérios empiricamente verificáveis que se relacionam tanto ao conhecimento ativo quanto ao conhecimento passivo do vocabulário. Além disso, os critérios visam, especificamente, a avaliação da profundidade do vocabulário utilizado pelos candidatos ao tratar do problema proposto pela tarefa metalinguística do exame. As delimitações realizadas seguem orientações de Milton (2009) para o estabelecimento de um foco avaliativo. Dessa forma, objetivou-se desenvolver uma escala que avalia a precisão do vocabulário utilizado pelos candidatos em sua produção oral.

Além disso, considerando as dimensões da avaliação do vocabulário de Read (2000), é possível dizer que o construto da precisão do vocabulário é integrado a um construto maior da proficiência oral do professor de LE - a expressão de conhecimento linguístico, uma vez que a escolha das palavras realizada pelo candidato reflete a sua habilidade de explicar a LE na língua-alvo (FREEMAN et al., 2015). Além disso, o construto operacionalizado é compreensivo, pois considera o input presente na tarefa metalinguística, assim como é dependente do contexto, uma vez que a produção oral se relaciona a uma tarefa que simula uma situação real de interação entre professor e aluno.

Para a classificação dos desempenhos mais e menos proficientes, foi tomado como critério principal o estabelecimento de relações coerentes entre terminologia técnica e/ou palavras referentes ao problema linguístico presentes na produção oral dos candidatos. Conforme apontado por Colombo (2019), parte das amostras de desempenho não apresentam emprego de metalinguagem, porém contém uma série 
de palavras e referentes os quais se relacionam ao problema linguístico proposto pela tarefa, sendo possível, dessa forma, verificar a coerência da explicação por meio da análise da precisão das palavras utilizadas pelos candidatos.

O Excerto 2, a seguir, apresenta a transcrição da amostra de desempenho oral do candidato TAC, em que se podem observar associações incoerentes entre a terminologia técnica empregada e as demais palavras escolhidas pelo candidato para abordar o problema linguístico proposto pela tarefa. Nota-se que, dessa forma, a precisão do vocabulário reflete um construto maior: o conhecimento do candidato acerca do tópico linguístico abordado. Dessa forma, evita-se que candidatos que expressam desconhecimento linguístico, mas empregam terminologia técnica, sejam classificados nas faixas mais altas da escala - uma possível solução para a questão levantada por Colombo (2019).

Excerto 2 - Desempenho oral do candidato TAC.

*RIC: let's go .

${ }^{*}$ RIC: on $\left[:\right.$ in] $\left[{ }^{*}\right]$ situation $B($.$) the best alternative is letter B$.

*RIC: you can $\mathrm{n}(\mathrm{o}) \mathrm{t}($.$) use the alternative \mathrm{A}($.$) because (.) the (.) verb$ tenses in the second sentence is wrong .

*RIC: if you (.) use "she might be away" (.) you have to change the tense "you: never know xxx her" to: another thing to [/] to say .

*RIC: this is completely unsense [//] (.) nonsense .

${ }^{*} \mathrm{RIC}$ : but (.) alternative B is correct .

*RIC: if you (.) really wanted to use the alternative A (.) \&yo you could (.) say: .

*RIC: she might be away (.) you [/] you never know about that .

\section{Fonte: o autor.}

À vista disso, por se tratar de uma tarefa metalinguística, o emprego preciso de terminologia técnica foi tomado como critério para delimitar as faixas superiores da escala de proficiência linguística. Uma vez que a tarefa número cinco do EPPLE aborda um problema linguístico e também orienta o candidato a empregar metalinguagem, o uso preciso de terminologia técnica sugere que o candidato possui níveis de 
proficiência mais altos. Além disso, incorporar o emprego de metalinguagem no quadro de critérios avaliativos também reflete o construto do conhecimento específico necessário ao professor de LE para ensinar a língua-alvo (ELDER, 2001; FREEMAN et al., 2015).

O Excerto 3, a seguir, apresenta a transcrição do desempenho oral do candidato LAC, que emprega terminologia técnica de maneira precisa, assim como estabelece uma relação coerente entre os termos técnicos utilizados e seus referentes para abordar o problema linguístico proposto pela tarefa metalinguística.

Excerto 3 - Desempenho oral do candidato LAC.

${ }^{*}$ LAC: so (.) \&i the sentence you would like to correct is .

*LAC: "if I happen to run into her I would tell her where you live" .

*LAC: right?

${ }^{*}$ LAC: the problem you (a)re having here (.) is: basically just (.) using the correct verb form .

"LAC: so if you say "if I happen" (.) you (a)re using the: present form .

${ }^{*} \mathrm{LAC}$ : and it refers to future.

"LAC: so you have to say "I will tell her where you live" .

"LAC: becau:se you can n(o)t (.) mix "if I happen" with "would" .

"LAC: if you prefer to use would conversely you can also say .

"LAC: "if I happened to run into her I would tell her where you live" .

*LAC: but then there is a slight difference (.) in that .

"LAC: when you say "if I happen" <it (i)s \&m> [//] it (i)s something that is more likely to occur .

"LAC: and if you say "if I happened" then: the likelihood is a bit lower .

*LAC: so that (i)s the basic difference .

Fonte: o autor.

Para os níveis inferiores, além da incoerência entre as palavras escolhidas para abordar o tópico linguístico proposto, a habilidade de reconhecer palavras-chave importantes relacionadas ao problema gramatical foi tomada como critério limitador entre as faixas mais baixas da escala. Foi possível observar que um número expressivo de candidatos não demonstrou conhecimento acerca das palavras presentes nos 
tópicos propostos, assim como desconheciam phrasal verbs empregados como distratores, provendo uma explicação incorreta e pouco satisfatória em vista do objetivo da tarefa.

O Excerto 4, a seguir, apresenta a transcrição do desempenho oral do candidato TAC, em que o examinando emprega corretamente terminologia técnica, assim como identifica palavras-chave necessárias para abordar o problema linguístico proposto, porém expressa desconhecimento acerca do tópico, provendo uma explicação imprecisa e, como consequência, teve seu desempenho classificado como insatisfatório.

Excerto 4 - Desempenho oral do candidato TAC.

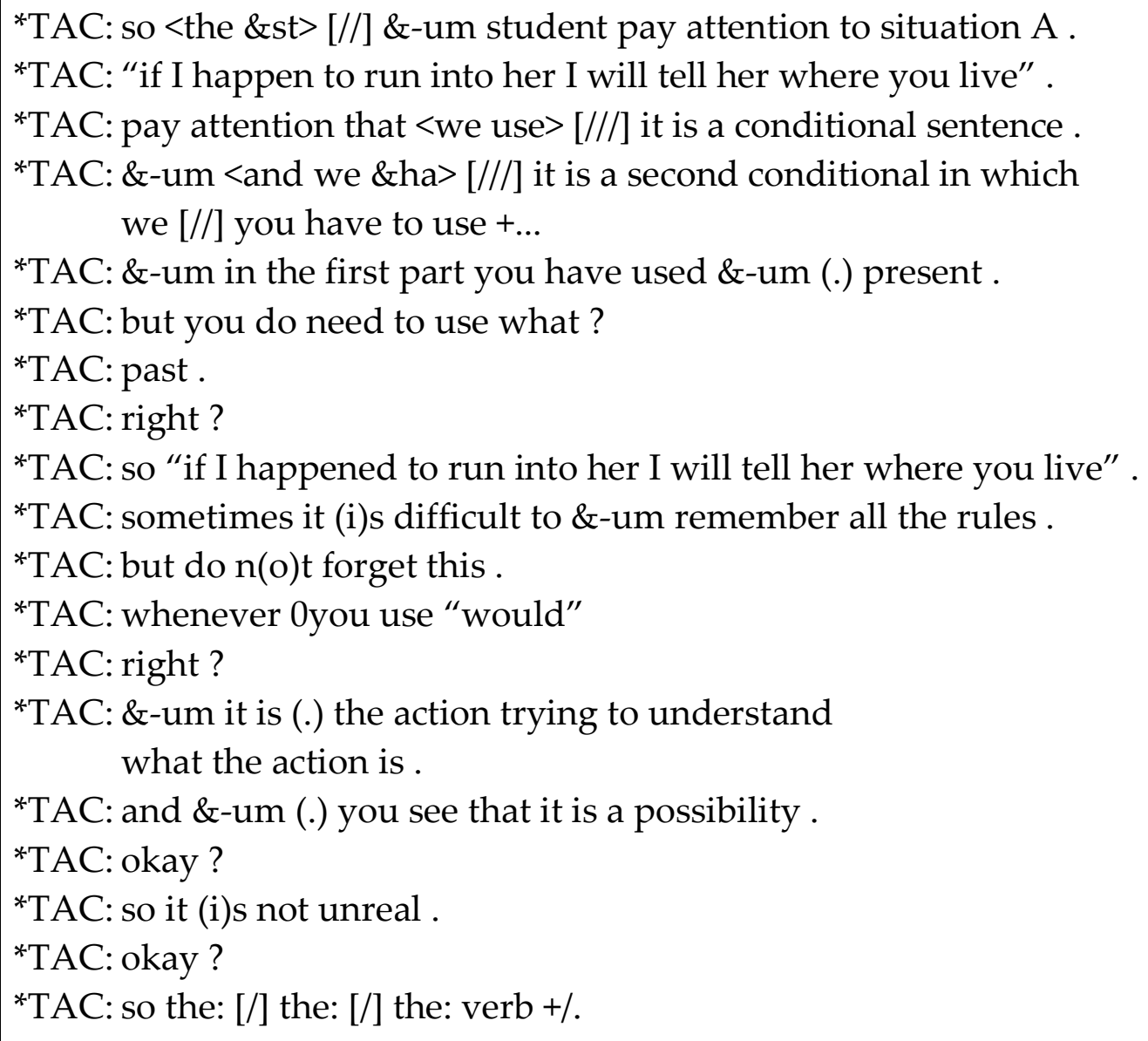


Os candidatos que expressaram desconhecimento de vocabulário em sua produção oral (conhecimento ativo), como também na interpretação da tarefa (conhecimento passivo), foram classificados na faixa mais baixa da escala, por apresentarem um desempenho extremamente insatisfatório. O Excerto 5, a seguir, apresenta a transcrição do desempenho oral do candidato LID que, ao reconhecer erroneamente a sentença you never know with her como agramatical, desenvolve uma explicação incoerente, imprecisa e que expressa desconhecimento em relação ao tópico abordado pela tarefa metalinguística.

Excerto 5 - Desempenho oral do candidato LID.

*LID: so: (.) I chose [: choose] [*] situation B .

*LID: \&-um what do you have to do here?

*LID: read letter A and then letter B .

*LID: "she might be away (.) you never know with her" .

*LID: in this situation you are using the wrong preposition .

*LID: so "with" and "her" is [: are] [*] not in the context.

*LID: you never know (.) about her .

*LID: you would never know about her .

*LID: you should use (.) another expression (.) to: (.) mean what you

(.) want to day .

*LID: letter B is better .

*LID: "she must be in Salvador (.) you never know" .

*LID: "you never know" because you do not know where (.) this person is where Mary is .

*LID: so: (.) in letter A (.) "you never know with her" (.) the preposition (.) is being used wrongly .

*LID: while in (.) letter B (.) "you never know" (.) is better .

*LID: because you do not have a preposition .

*LID: and you do not have (.) an undirect [: indirect]

$\left[{ }^{*}\right]$ object in your way .

Fonte: o autor.

Dessa maneira, é possível dizer que o estabelecimento de uma relação de coerência entre as palavras empregadas na explicação dos candidatos é o que determina, em primeiro lugar, o seu grau de precisão. Além disso, os desempenhos 
observados e classificados como mais precisos também apresentaram emprego adequado de metalinguagem, o que caracteriza o desempenho mais proficiente entre as amostras analisadas, por serem extremamente precisos quanto ao uso de terminologia técnica e expressarem conhecimento de vocabulário.

Nos desempenhos menos satisfatórios, ou seja, aqueles que apresentam incoerências entre as palavras empregadas para o provimento da explicação, o reconhecimento de palavras-chave para a resolução do problema gramatical foi tomado como critério que limita as faixas mais baixas da escala e que caracteriza o desempenho menos proficiente entre as amostras analisadas, por serem extremamente imprecisos quanto ao uso de terminologia técnica e expressarem desconhecimento de vocabulário.

A Figura 4, a seguir, ilustra a Árvore de Decisão de Desempenho e a escala de proficiência linguística desenvolvida para a avaliação da precisão do vocabulário com base em amostras de desempenho oral de candidatos do EPPLE.

Figura 4 - Árvore de Decisão de Desempenho e escala de proficiência linguística para a avaliação da precisão do vocabulário.

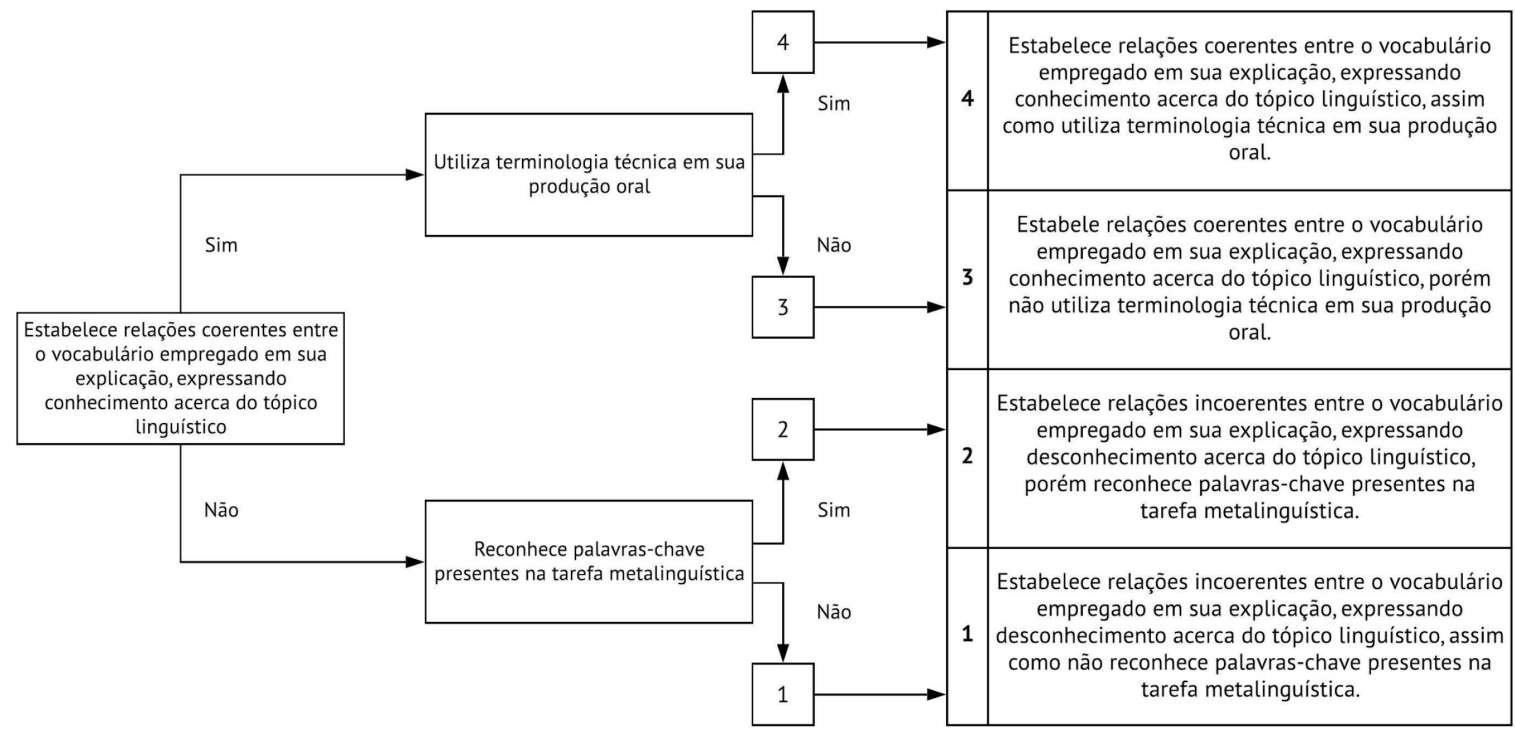

Fonte: o autor. 


\section{Considerações finais}

A partir da aplicação dos EBBs, foi possível desenvolver uma escala de proficiência linguística analítica para a avaliação da precisão do vocabulário empregado pelos candidatos do EPPLE, assim como a construção de um quadro de critérios empiricamente verificáveis os quais constituem um argumento para a validade do construto operacionalizado. Além disso, procurou-se contemplar os questionamentos levantados por Colombo (2019) em relação à escala de proficiência holística existente no EPPLE e apontamentos de Consolo e Teixeira da Silva (2014) acerca de aprimoramentos necessários ao exame, como, por exemplo, o desenvolvimento de uma escala analítica empiricamente embasada.

A escala de proficiência linguística produzida para a avaliação do vocabulário foca a profundidade do vocabulário do candidato (MILTON, 2009) e operacionaliza a precisão do vocabulário como dependente do contexto, integrado ao construto do conhecimento linguístico do professor de LE. Além disso, a escala caracteriza-se como compreensiva, uma vez que o desempenho dos candidatos é estritamente relacionado à tarefa metalinguística. Dessa forma, considerando as categorias avaliativas propostas por Read (2001), os critérios avaliativos contemplam o conhecimento de vocabulário ativo e passivo, os quais estão ligados tanto à compreensão do significado das palavras quanto ao seu uso na produção oral.

Foi possível observar que o estabelecimento de relações coerentes entre as palavras empregadas pelos candidatos em sua produção oral é o que caracteriza os desempenhos mais e menos proficientes. Para limitar os desempenhos mais proficientes, o emprego de terminologia técnica associada à expressão de conhecimento linguístico foi o critério tomado como definidor entre as fronteiras dos níveis superiores da escala. Para limitar os desempenhos menos proficientes, o desconhecimento acerca do significado de palavras-chave para abordar os tópicos 
linguísticos propostos pelo exame foi selecionado como critério limitador das faixas mais baixas da escala.

Sugere-se que investigações sejam realizadas para a compreensão de outras dimensões avaliativas do vocabulário empregado pelo professor de LE, como a sua abrangência, por exemplo. Além disso, é preciso que outros avaliadores/especialistas em avaliação empreguem a escala de proficiência produzida por este estudo em amostras de desempenho oral para que correlações possam ser estabelecidas entre os resultados obtidos e possíveis discrepâncias sejam identificadas. Tais procedimentos metodológicos assegurariam a confiabilidade dos resultados alcançados através desta pesquisa, assim como contribuiriam como argumento para a validade do construto operacionalizado na escala de proficiência analítica.

\section{Referências}

ALMEIDA FILHO, J. C. O professor de língua estrangeira sabe a língua que ensina? A questão da instrumentalização lingüística. Contexturas. Ensino Crítico de Língua Inglesa. UNICAMP, 1992.

COLOMBO, C. S. Avaliação de Proficiência Oral em Língua Estrangeira: Uma Proposta de Abordagem Avaliativa em um Exame para Professores de Línguas com base em Métodos de Estruturação de Problemas e em Métodos Multicritério de Análise da Decisão. 2019. Tese (Doutorado em Estudos Linguísticos) - Universidade Estadual Paulista "Júlio de Mesquita Filho". São José do Rio Preto/SP, 2019.

CONSOLO, D. A.; TEIXEIRA DA SILVA, V. L. Em defesa de uma formação linguística de qualidade para professores de línguas estrangeiras: o exame EPPLE. Revista Horizontes de Linguística Aplicada, v. 13, 2014. DOI https://doi.org/10.26512/rhla.v13i1.1334

CONSOLO, D. A; AGUENA, D. M. Pré-testagem ao Exame de Proficiência para professores de língua estrangeira (EPPLE): desenho e desafios tecnológicos para avaliação de proficiência oral em dispositivos tecnológicos móveis. Revista Polifonia, v. 24 n. 32/5, 2017. 
CONSOLO, D. A. Assessing EFL teachers' proficiency: on the development of teacher programmes and testing policies in Brazil. In: DOUGLAS A. C. ET AL (org). Perspectivas em avaliação no ensino e na aprendizagem de línguas: pesquisas e encaminhamentos na formação e na prática docente. Pontes Editores, Campinas, 2017.

COUNCIL OF EUROPE. Common European Framework of Reference for Languages, Learning, Teaching and Assessment. Strasbourg: Council of Europe Publishing. 2020. Disponível em www.coe.int/lang-cefr, acesso em 17/02/2021.

DALLER, H.; MILTON, J.; TREFFERS-DALLER, J. Conventions and terminology. In: DALLER, H.; MILTON, J.; TREFFERS-DALLER, J. (ed.). Modelling and Assessing Vocabulary Knowledge. Cambridge: Cambridge Applied Linguistics. 2007. DOI https://doi.org/10.1017/CBO9780511667268

ELDER, C. Assessing the language proficiency of teachers: are there any border controls? Language Testing, 2001, p. 149-170. DOI https://doi.org/10.1177/026553220101800203

FREEMAN, D.; KATZ, A.; GOMEZ; P. G.; BURNS, A. English-for-teaching: rethinking teacher proficiency in the classroom. Oxford: ELT Journal, Oxford University Press, 2015. DOI https://doi.org/10.1093/elt/ccu074

FULCHER, G.; DAVIDSON F.; KEMP, J. Effective rating scale development for speaking tests: Performance decision trees. Language Testing, 2011. DOI https://doi.org/10.1177/0265532209359514

FULCHER, G. Defining the construct. In: Testing second language speaking. New York: Routledge, 2014. DOI https://doi.org/10.4324/9781315837376

MILTON, J. Measuring Second Language Vocabulary Acquisition. Dublin: Trinity College. 2009. DOI https://doi.org/10.21832/9781847692092

NATION, I. S. P. Learning vocabulary in another language. Cambridge: Cambridge University Press, 2001. DOI https://doi.org/10.1017/CBO9781139524759

NICHOLLS, S. M. Aspectos pedagógicos e metodológicos do ensino de inglês. Maceió: EdUFAL, 2001.

READ, J. Assessing vocabulary. Cambridge: Cambridge University Press, 2000. DOI https://doi.org/10.1017/CBO9780511732942 
RODRIGUES, L. C. B. A formação do professor de língua estrangeira no século XXI: Entre as antigas pressões e os novos desafios. Revista Signum. 2016. DOI https://doi.org/10.5433/2237-4876.2016v19n2p13

SANDEI, M. L. R. Prospecções sobre a abordagem/senso de plausibilidade na ação de ensinar de um professor de língua estrangeira no ensino médio. 2005. Dissertação (Mestrado em Linguística Aplicada) - Universidade Estadual de Campinas, Campinas.

UPSHUR J. A; TURNER, C. E. Systematic effects in the rating of second-language speaking ability: test method and learner discourse. Language Testing. 1999. DOI https://doi.org/10.1177/026553229901600105

Anexo 1 - Códigos empregados na transcrição das amostras de desempenho oral.

\begin{tabular}{|c|c|}
\hline CÓDIGO & CARACTERISTICAS DE FALA \\
\hline "ABC: bla & Fala do interagente \\
\hline bla. & Declaraçāo completa \\
\hline bla $+\ldots$ & Declaração incompleta (sem interrupção) \\
\hline bla +1. & Declaração interrompida \\
\hline bla! & Exclamação completa \\
\hline bla? & Pergunta completa \\
\hline bla +..? & Pergunta incompleta (sem interrupção) \\
\hline bla +/? & Pergunta interrompida \\
\hline+ , bla & Auto-preenchimento (após interrupçāo) \\
\hline ++bla & Preenchimento do enunciado de outro falante \\
\hline "bla" & Citaçāo de pequenos trechos \\
\hline bla +"\%. & Citação longa iniciada na próxima trilha (tier) \\
\hline +" bla & Inicio de citação longa \\
\hline goed ["] [: went] & Erro estrutural seguido de correção pela transcrição \\
\hline piece [: peach] ["] & Erro de escolha lexical seguido de correção pela transcriçāo \\
\hline$<b l a>$ [] bla & Repetição de palavras ou trechos de fala \\
\hline$\langle$ bla $\rangle[/]$ blabla & Reconstituiçâa de palavras ou trechos de fala \\
\hline$\langle$ bla $>$ [H] blabla & Falso inicio sem reconstituição \\
\hline$\langle b l a>[I / I]$ cha & Reformulação completa da mensagem \\
\hline bla ["][] bla & Erro no trecho de fala a ser reparado \\
\hline bla [] ["] bla & Erro no trecho de fala de reparo \\
\hline Obla & Palavra omitida \\
\hline bl(a) & Encurtamento fonológico \\
\hline bla:bla & Alongamento de som silábico \\
\hline blabla: & Alongamento de palavra \\
\hline \&bl & Fragmentos fonológicos \\
\hline \&-um & Pausas preenchidas (preenchedores não lexicais) \\
\hline bla^bla & Pausa dentro de uma palavra \\
\hline (.) & Pausa silenciosa (sem duração) \\
\hline (0.6) & Pausa silenciosa (com duração em segundos) \\
\hline$x x x$ & Discurso incompreensivel \\
\hline bla [=! tosse] & Vocalizaçōes não verbais (ocorrência paralinguistica) com ocorrência linguistica na trilha (tier) \\
\hline $0[=!$ tosse $]$ & Vocalizaçōes não verbais (ocorrência paralinguistica) sem ocorrência linguistica na trilha (tier) \\
\hline bla $\uparrow$ & Entonaçä́o ascendente \\
\hline bla $\downarrow$ & Entonaçāo descendente \\
\hline bla \&*ABC: bla bla & Sobreposição de discurso (comentários curtos) \\
\hline $\begin{array}{l}<b \mid a>[>] \\
\langle b| a>[<]\end{array}$ & Sobreposição de discurso (falas simultâneas) \\
\hline bla@s:por & Palavra em lingua portuguesa \\
\hline bla@q & Uso de metalinguagem \\
\hline \%pho: & Desvio de pronúncia \\
\hline bla'bla & Silaba enfatizada \\
\hline bla <bla bla> [!] & Trecho enfatizado \\
\hline \%com: bla & Comentários \\
\hline bla $\ddagger$ bla & Agrupamento de marcadores conversacionais (posiçāo inicial): vocativos \\
\hline bla , bla & Agrupamento de marcadores conversacionais (posiçāo final): vocativos, perguntas curtas \\
\hline bla [= bla] & Identidade dếtica \\
\hline blat. & Pausa na transcrição \\
\hline bla $\left[{ }^{\wedge} \mathrm{c}\right]$ & Delimita orações dentro de sentenças complexas \\
\hline
\end{tabular}

Fonte: Colombo (2019). 